TSUKUBA J. MATH.

Vol. 8 No. 1 (1984). $159-170$

\title{
THE APPROXIMATE SECTION EXTENSION PROPERTY AND HEREDITARY SHAPE EQUIVALENCES ${ }^{(1)}$
}

\author{
By
}

\author{
Tatsuhiko YAGASAKI
}

\begin{abstract}
In this paper, the concept of the approximate section extension property (ASEP) is introduced. It is shown that hereditary shape equivalences are exactly the maps with the hereditary ASEP and every $\mathrm{UV}^{n-1}$-map with $n$-dimentional range has the ASEP.
\end{abstract}

\section{Introduction.}

In this paper we will introduce the concept of the approxsmate section extension property (ASEP), which is a shape version of the section extension property, [Do]. The ASEP is defined in Section 1 to maps between metric spaces. However, using resolutions of maps, this can be extended to a general case (see Section 3). Main results of the paper are contained in Section 2. We prove the followings:

1) Hereditary shape equivalences (HSE's) are exactly the maps with the hereditary ASEP. In particular, any pull back of a HSE is also HSE.

2) Every $\mathrm{UV}^{n-1}$-map with an $n$-dimensional range and every $\mathrm{UV}^{\infty}$-map between ANR's has the ASEP. If the range is a manifold, then an appropriate converse holds.

One can regard these results as a shape version of some results in the fiber homotopy theory, [Do].

Here, we list some notations to be used throughout the paper. In Sections 1 and 2, spaces are assumed to be metrizable. If $A$ is a subset of a space $X, \bar{A}$ is the closure of $A$ and inc $(A, X)$ denotes the inclusion map $A \subset X$. Cov $X$ is the set of all normal coverings of $X$. For $\varnothing \in \operatorname{Cov} Y$, st $\varnothing$ is the star of $\varnothing$. We say two maps $f, g: X \rightarrow Y$ are $\downarrow$-near and write $(f, g) \leq \mathcal{}$ when each $x \in X$ admits a $V \in C V$ such that $f(x), g(x) \in V$. An ANR is one for metric spaces. A polyhedron is the body $|K|$ of a simplicial complex $K$ with the $C W$-topology.

We refer to [DS] for the definitions of basic terms in Shape theory, and to [A1] for relation theoretic terms. One should refer to [Do] in Sections 1,2 and to [Ma] in Section 3.

(1) This work is partially included in the author's master thesis.

Received August 24, 1983. 


\section{Approximate section extension property.}

Let $f: X \rightarrow Y$ be a map between metric spaces. We can find closed embeddings $i: X \rightarrow M, j: Y \rightarrow N$ into ANR's and a map $F: M \rightarrow N$ with $F i=j f([\mathrm{H}])$. Consider a pair $(U, V)$ of open neighborhoods (nbd's) $U$ of $i(X)$ in $M$ and $V$ of $j(Y)$ in $N$ with $F(U) \subset V$. We call such a pair an admissible pair for $f$ w. r.t. $F$.

PROPOSITION 1.1: Under the above notations, the following conditions (1)-(3) are equivalent. Furthermore if the map $f$ satisfies the condition (1) (eq., (2), (3)), for some $i, j$ and $F$ as above, then $f$ satisfies the condition (1) for any such $i, j$ and $F$.

(1) For each admissible pair $(U, V) \quad \in \in \operatorname{Cov} U$ and $\propto \mathcal{V} \in \operatorname{Cov} V$, there xeist an admissible pair $\left(U_{1}, V_{1}\right) \geq(U, V)$ (i.e., $\left.U_{1} \subset U, V_{1} \subset V\right)$ and $C_{1} \in \operatorname{Cov} V_{1}$ such that for each closed set $A$ of $V_{1}$ and each map $s: A \rightarrow U_{1}$ with $\left(F s\right.$, inc $\left.\left(A, V_{1}\right)\right) \leq V_{1}$, there exist an open nbd $W$ of $j(Y)$ in $V_{1}$ and a map $S: W \rightarrow U$ with $(F S$, inc $(W, V)$ ) $\leq \mathcal{V}$ and $\left(\left.S\right|_{A \cap W},\left.s\right|_{A \cap W}\right) \leq \mathcal{U}$.

(2) For each admissible pair $(U, V), \quad U \in \operatorname{Cov} U$ and $\subset \in \operatorname{Cov} V$, there exist $\left(U_{1}, V_{1}\right) \geq(U, V)$ and $\mathcal{V}_{1} \in \operatorname{Cov} V_{1}$ such that for each closed set $A$ of $Y$ and each map $s: A \rightarrow U_{1}$ with (Fs, inc $\left.\left(A, V_{1}\right)\right) \leq \mathcal{V}_{1}$, there exists a map $S: Y \rightarrow U$ with (FS, $\operatorname{inc}(Y, V)) \leq C$ and $\left(\left.S\right|_{A}, s\right) \leq \mathcal{U}$.

(3) For each open nbd $U$ of $i(X)$ in $M$ and $\subset \in \operatorname{Cov} N$, there exist an open nbd $U_{1}$ of $i(X)$ in $U$ and $\mathcal{V}_{1} \in \operatorname{Cov} N$ such that for each closed set $A$ of $Y$ and each map $s: A \rightarrow U_{1}$ with $(F s$, inc $(A, N)) \leq V_{1}$, there exists a map $S: Y \rightarrow U$ with $(F S, \operatorname{inc}(Y, N)) \leq C$ and $\left.S\right|_{A}=s$.

Proof: Note that $U$ and $U_{1}$ are ANR's. Therefore $(2) \rightarrow(1)$ follows from the nbd extension property of ANR's and $(2) \rightarrow(3)$ follows from the hom otopy extension theorem $([\mathrm{H}]]) . \quad(1) \rightarrow(2)$ and $(3) \rightarrow(2)$ are obvious. For the latter statement, see Proposition 3.2, which implies the same conclusion under a more general setting.

DEFINITION 1.2: We say the map $f$ has the approximate section extension property (ASEP) provided $f$ satisfies the conditions in Proposition 1.1.

If $f$ is a proper map (i. e., the inverses of compact sets are compact), then we can reduce the above conditions to a simpler one. For later use, we shall work in the setting of relation.

Suppose $M$ and $N$ are ANR's, $Y$ is a closed subset of $N, R: Y \rightarrow M$ is an (upper semi-) continuous relation with compact point images (i. e., for each $y \in Y$, $R(y)$ is compact) ([A1]) and $p: N \times M \rightarrow N$ is the projection. Note that $p(R) \subset Y$. 
Propositjon 1.3: Under the above notations, the projection $p: R \rightarrow Y$ has the $A S E P$ iff

(\#): each nbd $U$ of $R$ in $Y \times M$ contains a nbd $V$ of $R$ such that

$\left(^{*}\right)$ : for each closed set $A$ of $Y$ and each map $s: A \rightarrow V$ with $p s=\operatorname{inc}(A, Y)$, there exists a map $S: Y \rightarrow U$ with $p S=1_{Y}$ and $\left.S\right|_{A}=s$.

Let $f: X \rightarrow Y$ be a proper map and $M$ an ANR containing $X$ as a closed subset. Consider the continuous relation $f^{-1}=\cup\left\{y \times f^{-1}(y): y \in Y\right\}: Y \rightarrow M$. Since the projection $p: f^{-1} \rightarrow Y$ corresponds to $f$ by the identification $f^{-1} \rightarrow X:(f(x), x) \mapsto x$, we get the following.

COROLLARY 1.4: Under the above notation, $f$ has the ASEP iff (\#) holds with $R$ replaced by $f^{-1}$.

REMARK 1.5: The map $f$ is said to be approximately invertible ([A2]) if each nbd $U$ of $f^{-1}$ in $Y \times M$ admits a map $S: Y \rightarrow U$ such that $p S=1_{Y}$.

Proof of 1.3: From Proposition 1.1, $p$ has the ASEP iff

(\#\#): for each nbd $U^{\prime}$ of $R$ in $N \times M$ and $U \in \operatorname{Cov} N$, there exist a nbd $V^{\prime}$ of $R$ in $U^{\prime}$ and $Q \in \operatorname{Cov} N$ such that

$\left({ }^{* *}\right)$ : if $A$ is a closed set of $Y$ and $s^{\prime}: A \rightarrow V^{\prime}$ is a map with $\left(p s^{\prime}\right.$, inc $\left.(A, N)\right)$ $\leq \mathcal{V}$, then there exists $S^{\prime}: Y \rightarrow U^{\prime}$ with $\left(p S^{\prime}\right.$, inc $\left.(Y, N)\right) \leq \mathcal{U}$ and $\left.S^{\prime}\right|_{A}=s^{\prime}$.

$(\#) \rightarrow(\# \#)$ : Given $U^{\prime}$ and $U$ as in (\#\#). By [A1], Lemma A-8, there exist an open nbd $U_{1}^{\prime}$ of $R$ in $U^{\prime}$ and $U_{1}=\left\{U_{k}\right\}_{\kappa \in K} \in \operatorname{Cov} N$ such that $U_{\kappa} \times U_{1}^{\prime}\left(U_{\kappa}\right) \subset U^{\prime}$ for each $\kappa \in K$ and $U_{1}<U$. Apply (\#) to $U=U_{1}^{\prime} \cap(Y \times M)$ and we have an open nbd $V$ of $R$ in $U$ which satisfies (*). By [A1], Lemma A-8, there exist a nbd $V^{\prime}$ of $R$ in $U_{1}^{\prime}$ and a $\varnothing=\left\{V_{\lambda}\right\}_{\lambda \in A} \in \operatorname{Cov} N$ such that $\left(V_{\lambda} \times V^{\prime}\left(V_{\lambda}\right)\right) \cap(Y \times M) \subset V$ for each $\lambda \in \Lambda$ and $\varnothing$-near maps to $N$ are $U_{1}$-homotopic. Then $V^{\prime}$ and $Q$ satisfy (**). In fact, take $s^{\prime}$ as in (**). Since $\left(p s^{\prime}\right.$, inc $\left.(A, N)\right) \leq q$, we can define $s: A \rightarrow V$ by $s(y)=\left(y, \pi s^{\prime}(y)\right)$ $(y \in A)$, where $\pi: N \times M \rightarrow M$ is the projection. By $\left(^{*}\right)$, the map $s$ extends to a map $S: Y \rightarrow U$ with $p S=1_{Y}$, and since $p s^{\prime}$ is $U_{1}$-homotopic to inc $(A, N)$, using the homotopy extension theorem, $p s^{\prime}$ extends to a map $g: Y \rightarrow N$ which is $\mathcal{U}_{1}$-homotopic to inc $(Y, N)$. Then the desired map $S^{\prime}: Y \rightarrow U^{\prime}$ is defined by $S^{\prime}(y)=(g(y), \pi S(y))$ $(y \in Y)$.

$(\# \#) \rightarrow(\#)$ : The proof is similar and omitted.

We use 1.4 to obtain the Uniformization Theorem for the ASEP. Compair this with [Do], Theorem 2.7.

THEOREM 1.6: Suppose $f: X \rightarrow Y$ is a proper map. If each $y \in Y$ admits a 
(not necessarily open) nbd $V$ in $Y$ such that $\left.f\right|_{f^{-1}(V)}: f^{-1}(V) \rightarrow V$ has the $A S E P$, then $f$ itself has the ASEP.

Proof: Consider the following property $\mathscr{P}(A)$ for each subset $A$ of $Y$.

$\mathscr{L}(A): f_{A}$ has the ASEP, where $f_{A}=\left.f\right|_{f^{-1}(A)}: f^{-1}(A) \rightarrow A$. In order to show $\mathscr{Q}(Y)$ holds, by [Mi], Theorem 5.5 , it suffices to show that $\mathscr{P}$ is an F-hereditary property, that is, satisfies the following conditions:

(F1) If $f_{A}$ has the ASEP and $B$ is a closed subset of $A$, then $f_{B}$ has the ASEP.

(F2) Suppose $A=A_{1} \cup A_{2} \subset Y$ and $A_{1}$ and $A_{2}$ are closed in $Y$. If $f_{i}=f_{A_{i}}$ has the $A S E P(i=1,2)$, then $f_{A}$ has the ASEP.

(F3) Suppose $A=\cup\left\{A_{\lambda}: \lambda \in \Lambda\right\} \subset Y$ and $\left\{A_{\lambda}\right\}_{\lambda \in \Lambda}$ is discrete in $Y$. If each: $f_{A_{\lambda}}$ has the ASEP, then $f_{A}$ has the ASEP.

(F1) and (F3) are easily verified, so we will prove (F2). We use the same notation as in Corollary 1.4. Note that $f_{i}^{-1}=f^{-1} \cap\left(A_{i} \times M\right)(i=1,2)$. To see the ASEP of $f_{A}$, let $U$ be any open nbd of $f_{A}^{-1}$ in $A \times M$. Since $f_{1}$ has the ASEP, the nbd $U \cap\left(A_{1} \times M\right)$ of $f_{1}^{-1}$ contains an open nbd $V_{1}$ of $f_{1}^{-1}$ in $A_{1} \times M$ which satisffes the condition $\left(^{*}\right)$ in Proposition 1.3 w. r.t. $f_{1}^{-1}$. In turn, by the ASEP of $f_{2}$, the open nbd $U_{2}=\left\{V_{1} \cup\left(U-\left(A_{1} \times M\right)\right)\right\} \cap\left(A_{2} \times M\right)$ of $f_{2}^{-1}$ in $A_{2} \times M$ contains an open nbd $V_{2}$ of $f_{2}^{-1}$ in $A_{2} \times M$ which satisfies $\left(^{*}\right)$ w.r.t. $f_{2}^{-1}$. Then the nbd $V=\left(V_{1}-\right.$ $\left.\left(A_{2} \times M\right)\right) \cup V_{2}$ of $f^{-1}$ in $U$ satisfies $\left(^{*}\right)$ w. r. t. $f^{-1}$ and $U$.

\section{The ASEP, HSE's and UV ${ }^{n}$-maps.}

We begin with a reference to [A1], Let $f: X \rightarrow Y$ be a proper map between metric spaces, and $M$ an ANR containing $X$ as a closed subset.

THEOREM ([A1], Theorem 4.5): The map $f$ is a HSE iff the relation $f^{-1}: Y \rightarrow M$ is slice trivial, that is, satisfies the following:

For each nbd $U$ of $f^{-1}$ in $Y \times M$ there exist a nbd $V$ of $f^{-1}$ and maps $\phi: V \times$ $[0,1] \rightarrow U$ and $S: Y \rightarrow U$ such that $\phi_{0}=\operatorname{inc}(V, U), \dot{\phi}_{1}(y, x)=S(y), p \phi(y, x, t)=y, p S=1_{Y}$ $((y, x) \in V, t \in[0,1])$, where $p: Y \times M \rightarrow Y$ is the projection.

We will use the above theorem to get another characterization of HSE's in term of the ASEP, which corresponds to [Do], Proposition 3.1.

Let $\alpha: B \rightarrow Y$ is a proper map. Define $E$ and $f_{\alpha}: E \rightarrow B, \beta: E \rightarrow X$ by $E=\{(b, x)$ $\epsilon B \times X: \alpha(b)=f(x)\}, f_{\alpha}(b, x)=b, \beta(b, x)=x,((b, x) \in E)$. The map $f_{\alpha}$ is called the map induced from $f$ by $\alpha$. Since $E=\cup\left\{b \times f^{-1} \alpha(b): b \in B\right\}=\cup\left\{\alpha^{-1}(y) \times f^{-1}\left(y^{\prime}\right): y \in Y\right\}$, if we regard $E$ as a relation from $B$ to $X$, we have:

1) $E=f^{-1} \alpha$, therefore $E$ is continuous and has compact point images.

2) $\alpha \times 1_{M}: B \times M \rightarrow Y \times M$ is a closed map and $E=\left(\alpha \times 1_{M}\right)^{-1}\left(f^{-1}\right)$. 
LEMMA 2.1: Under the above notation, the following conditions are equivalent.

a) $f_{\alpha}$ has the ASEP.

b) Each nbd $U$ of $f^{-1}$ in $Y \times M$ contains a nbd $V$ of $f^{-1}$ such that if $A$ is a closed set of $B$ and $\alpha_{0}: A \rightarrow V$ is a map with $p \alpha_{0}=\left.\alpha\right|_{A}$, then there exists a map $\alpha^{\prime}$ : $B \rightarrow U$ with $p \alpha^{\prime}=\alpha$ and $\left.\alpha^{\prime}\right|_{A}=\alpha_{0}$.

Proof: By Proposition 1.3, a) is equivalent to 1.3 (\#), with $R$ and $Y$ replaced by $E$ and $B$. By the observation 2), $\alpha \times 1_{M}$ gives the correspondence between a nbd base of $f^{-1}$ in $Y \times M$ and a nbd base of $E$ in $B \times M$, so that the maps $\alpha_{0}$ and $\alpha^{\prime}$ as in b) correspond to the maps $s$ and $S$ as in (\#) and (*). From this follows 2.1. Compair this with [Do], Proposition 3.1.

Let $\tilde{f}$ be the map induced from $f$ by $f r$, i. e., $\tilde{f}=f_{f r}$, where $r: X \times[0,1] \rightarrow X$ is the projection.

THEOREM 2.2: Let $f: X \rightarrow Y$ be a proper map. The following conditions are equivalent.

a) For each proper map $\alpha: B \rightarrow Y, f_{\alpha}$ has the ASEP.

b) $f$ is approximately invertible (see Remark 1.5) and $\tilde{f}$ has the ASEP.

c) $f$ is a HSE.

d) Each nbd $U$ of $f^{-1}$ in $Y \times M$ contains a nbd $V$ of $f^{-1}$ such that for any proper map $\alpha: B \rightarrow Y$, Lemma 2.1, b) holdds.

Since $\left(f_{\alpha}\right)_{\beta}=f_{\alpha \beta}$ for any maps $C \stackrel{\beta}{\rightarrow} B \stackrel{\alpha}{\rightarrow} Y$, we have the following.

Corollary 2.3: If $f$ is a HSE, then $f_{\alpha}$ is a HSE for any proper map $\alpha$ : $B \rightarrow Y$.

REMARK 2.4: By [A1], Lemma 4.6, we see the relation $E=f^{-1} \alpha: B \rightarrow M$ is slice trivial if $f^{-1}: Y \rightarrow M$ is slice trivial. This also implies 2.3.

Proof of 2.2: (a) $\rightarrow$ (b) follows from 1.4. (b) $\rightarrow$ (c): It suffices to show that each open nbd $U$ of $f^{-1}$ in $Y \times M$ admits a slice contraction $\phi: f^{-1} \times[0,1] \rightarrow U$ of $f^{-1}$ in $U$. (See [A1], Lemma 4.3.) Applying Lemma 2.1 to $\tilde{f}$, we can find an open nbd $V$ of $f^{-1}$ in $U$ such that each map $g: X \times\{0,1\} \rightarrow V$ with $p g=\left.f r\right|_{X \times(0,1)}$ has an extension $G: X \times[0,1] \rightarrow U$ with $p G=f r$. Since $f$ is approximately invertible, there exists a map $S: Y \rightarrow V$ with $P S=1_{Y}$. Then the map $g: X \times\{0,1\} \rightarrow V$ defined by $g(x, 0)=$ $(f(x), x) \in f^{-1}$ and $g(x, 1)=S(f(x)) \in V(x \in X)$ admits an extension $G$ as above. Define $\phi: f^{-1} \times[0,1] \rightarrow U$ by $\phi(y, x, t)=G(x, t) \quad\left((y, x, t) \in f^{-1} \times[0,1]\right) . \quad(\mathrm{c}) \rightarrow(\mathrm{d}):$ See the proof of [A1], Proposition 2.2. (d) $\rightarrow$ (a) follows from Lemma 2.1. 
Next we study $U^{n}$-maps with the ASEP. We recall some definition:s of basic terms. For $n \geq 0, \boldsymbol{S}^{n}$ denotes the standard $n$-sphere and $\boldsymbol{B}^{n}$ denotes the $n$-ball. Suppose $X$ be a metric space and $i: X \rightarrow M$ is a closed embedding into an ANR $M$. We say $X$ is $\mathrm{UV}^{n}$ (or $\mathrm{AC}^{n}$ ) provided each nbd $U$ of $i(X)$ in $M$ contains a nbd $V$ of $i(X)$ such that every $\alpha: \boldsymbol{S}^{k} \rightarrow V(0 \leq k \leq n)$ is null homotopic in $U$. The definition does not depend on the choice of such an embedding $i . X$ is UV ${ }^{\infty}$ if $X$ is $\mathrm{UV}^{n}$ for each $n \geq 0$. A UV ${ }^{n}$-map $(0 \leq n \leq \infty)$ is an onto map each point inverse of which is $\mathrm{UV}^{n}$. For the details, see [Dy], [K] and [L]. We will prove the following theorem.

Theorem 2.5: Suppose $f: X \rightarrow Y$ is a closed onto map.

(1) If $f$ is $a \mathrm{UV}^{n-1}-m a p$ and $\operatorname{dim} Y \leq n<\infty$, then $f$ has the ASEP.

(2) If $f$ is $a \mathrm{UV}^{\infty}-m a p$ and $X$ and $Y$ are ANR's, then $f$ has the ASE'P.

The proof is based on the following lifting lemmas.

Lemma 1 ([Dy], Lemma 8.3): Under the same notation as in Proposition 1.1, suppose the map $f$ is a closed $\mathrm{UV}^{n-1}$-map $(0 \leq n<\infty)$. Then for each admissible pair $(U, V)$ for $f$ w.r.t. $F$ and each $\mathcal{V}^{\prime} \in \operatorname{Cov} V$, there exist $\left(U_{1}, V_{1}\right) \geq(U, V)$ and $Q_{1} \in \operatorname{Cov} V_{1}$ such that

$\left.{ }^{*}\right)$ if $(P, Q)$ is a polyhedral pair with $\operatorname{dim} p \leq n$ and $g: P \rightarrow V_{1}, h: Q \rightarrow U_{1}$ are maps with $\left(\left.g\right|_{Q}, F h\right) \leq V_{1}$, then there exists a map $g^{\prime}: P \rightarrow U$ with $\left.g^{\prime}\right|_{Q}=h$ and $\left(F g^{\prime}, g\right)$ $\leq \mathcal{V}^{\prime}$.

Lemma 2 ([K], Theorem 1, Part II): Let $f: X \rightarrow Y$ be an $\mathrm{LC}^{\infty}$-dense map. Then for each locally finite open covering $\subset$ of $Y$, polyhedral pair $(P, Q)$ and maps $g: P \rightarrow Y, h: Q \rightarrow X$ with $\left.g\right|_{Q} \simeq f h$ ( $\left(V\right.$-homotopic), there exists a map $g^{\prime}: P \rightarrow X$ with $\left.g^{\prime}\right|_{Q}=h$ and $f g^{\prime} \simeq g$ (st CV-homotopic).

REMARK 2.6: 1) Among closed onto maps with ANR domains, LC ${ }^{c o}$-(dense) maps coincide with $\mathrm{UV}^{\infty}$-maps.

2) Theorem 2.5, (2) holds even if $X$ is an approximate polyhedron (A]) ([Ma]) and $f$ is an $\mathrm{LC}^{\infty}$-dense map.

However, it will be shown that Taylor's map $F: X \rightarrow Q$ does not have the ASEP (Example 3.7). Hence we can't omit the assumption on $X$ if $\operatorname{dim} Y=\infty$.

Proof of 2.5: (1) Under the same notation as in Proposition 1.1, we will show that $f$ satisfies the condition (2) in 1.1. Given any admissible pair $(U, V)$, $U \in \operatorname{Cov} U$ and $\sigma \in \operatorname{Cov} V$. Let $V^{\prime}$ be a star refinement of $\sigma$. By Lemma 1, we get $\left(U_{1}, V_{1}\right) \geq(U, V)$ and $C_{1} \in \operatorname{Cov} V_{1}$ which satisfy $\left(^{*}\right)$. We must verify that 
$\left(U_{1}, V_{1}\right), C V_{1}$ satisfy the required condition in Proposition 1.1, (2) w. r.t. $(U, V)$, $U$ and $\checkmark$. Let $A$ be a closed set of $Y, W$ an open nbd of $A$ in $V_{1}$ and $s: W \rightarrow$ $U_{1}$ a map with $\left(F s\right.$, inc $\left.\left(W, V_{1}\right)\right) \leq V_{1}$. Take an open nbd $W_{1}$ of $A$ with $\bar{W}_{1} \subset W$ and a common refinement $\mathscr{W} \in \operatorname{Cov} V_{1}$ of coverings $\left.\mathcal{V}^{\prime}\right|_{V_{1}},\left\{V_{1}-A, W_{1}\right\}$ and $s^{-1}\left(\left.\mathcal{U}\right|_{U_{1}}\right)$ $\cup\left\{V_{1}-A\right\}$. Since $\operatorname{dim} Y \leq n$ and $V_{1}$ is an ANR, there exist a polyhedron $P$ and maps $Y \stackrel{i}{\rightarrow} P \stackrel{r}{\rightarrow} V_{1}$ such that $\operatorname{dim} P \leq n$ and $\left(r i, \operatorname{inc}\left(Y, V_{1}\right)\right) \leq \mathscr{W}([\mathrm{H}]$, Themrem 6.1). Choose a triangulation $K$ of $P$ such that $\{|\sigma|: \sigma \in K\}$ refines $\left\{r^{-1}(W), P-r^{-1}\left(\bar{W}_{1}\right)\right\}$, and put $Q=\cup\left\{|\sigma|: \sigma \in K,|\sigma| \cap r^{-1}\left(\bar{W}_{1}\right) \neq \emptyset\right\}$. Then $Q$ is a subpolyhedron of $P$ and $i(A)$ $\subset Q, r(Q) \subset W$. Since $\left(\left.F s r\right|_{Q},\left.r\right|_{Q}\right) \leq C V_{1}$, by $\left(^{*}\right)$, we obtain a map $r^{\prime}: P \rightarrow U$ with $\left(F r^{\prime}, r\right) \leq V^{\prime}$ and $\left.r^{\prime}\right|_{Q}=\left.s r\right|_{Q}$. Put $S=r^{\prime} i: Y \rightarrow U$, then it is easy to see that (FS, inc $(U, V)) \leq C$ and $\left(\left.S\right|_{A},\left.s\right|_{A}\right) \leq \mathcal{U}$. This completes the proof of (1).

(2) is verified by the same argument as in (1), using Lemma 2 instead of Lemma 1.

The next theorem is a partial converse of the above theorem.

THEOREM 2.7: Let $f: X \rightarrow Y$ be a proper (onto) map with the ASEP and $y \in Y$. If each nbd $V$ of $y$ in $Y$ contains an $n$-sphere contractible in $V$, then $f^{-1}(y)$ is $\mathrm{UV}^{n}$.

Proof. Take a closed embedding $X \subset M$ into an ANR $M$. To show $f^{-1}(y)$ is $\mathrm{UV}^{n}$ (in $M$ ), let $U_{1}$ be any open nbd of $f^{-1}(y)$ in $M$. We must find a nbd $U_{2}$ of $f^{-1}(y)$ in $U_{1}$ as in the definition of $U V^{n}$-property. Take an open nbd $V_{1}, V_{2}$ of $y$ in $Y$ with $f^{-1}\left(V_{1}\right) \subset U_{1}, \bar{V}_{2} \subset V_{1}$ and let $\tilde{U}=V_{1} \times U_{1} \cup\left(Y-\bar{V}_{2}\right) \times M$. Then by Corollary 1.4, we get an open nbd $\tilde{V}$ of $f^{-1}$ in $\tilde{U}$ which satisfies Proposition 1.3, (*). Take a nbd $V_{3}$ of $y$ in $V_{2}$ and a nbd $U_{2}$ of $f^{-1}(y)$ in $U_{1}$ such that $V_{3} \times U_{2} \subset \tilde{V}$. By the assumption, $V_{\mathbf{3}}$ contains an $n$-sphere $\boldsymbol{S}^{n}$ contractible in $V_{3}$. Now for $0 \leq i$ $\leq n$, consider $\boldsymbol{S}^{i} \subset \boldsymbol{S}^{n}$ and given any map $\alpha: \boldsymbol{S}^{i} \rightarrow U_{2}$. Then the map $s: \boldsymbol{S}^{i} \rightarrow \tilde{V}$ defined by $s(x)=(x, \alpha(x))\left(x \in \boldsymbol{S}^{i}\right)$ can be extended to a map $S: Y \rightarrow \tilde{U}$ with $p S=1_{Y}$. Since $\boldsymbol{S}^{i} \simeq 0$ in $V_{3}$, we have a map $h: \boldsymbol{B}^{i+1} \rightarrow V_{3}$ such that $\left.h\right|_{\boldsymbol{S}^{i}}=\operatorname{inc}\left(\boldsymbol{S}^{i}, Y\right)$. Then $\pi S h: B^{i+1} \rightarrow U_{1}$ is an extension of $\alpha$, where $\pi: Y \times M \rightarrow M$ is the projection. This completes the proof.

CoROllary 2.8: Let $f: X \rightarrow Y$ be a proper map.

(1) Suppose $\operatorname{dim} Y \leq n(0 \leq n<\infty)$ and each non-empty open set $U$ of $Y$ contains an $(n-1)$-sphere contractible in $U$. Then the map $f$ has the ASEP iff $f$ is a $\mathrm{UV}^{n-1}$. map

(2) Suppose $X$ and $Y$ are ANR's and each non-empty open set $U$ of $Y$ contains an $n$-sphere for each $n \geq 0$. Then $f$ has the ASEP iff $f$ is a $\mathrm{UV}^{\infty}$-map.

REMARK 2.9: If $\operatorname{dim} Y=n(0 \leq n<\infty)$ and $Y$ contains a dense open set which 
is an n-manifold, then $Y$ satisfies the condition in (1). If $Y$ is a $\boldsymbol{Q}$ (or $\alpha_{2}$ )-manifold, then $Y$ satisfies the condition in (2).

\section{Generalization.}

In this section, we extend the definition of the ASEP to the general case. The manner of the generalization has been established in [Ma], where the concept of shape fibrations is generalized using $(A P-)$ resolutions of maps. We shall show the same method works for the ASEP as well as shape fibrations.

We use the same notations as in [Ma]. In addition, $A(\underline{p})$ denotes the set of all admissible pairs of $a$ map $\underline{p}$ of systems. In this section, spaces are not assumed to be metrizable. Let $B$ be a space and $A \subset V$ subsets of $B$. By definition, $V$ is a halo of $A$ in $B$ if there is a map $\tau: B \rightarrow[0,1]$ with $A \subset \tau^{-1}(1)$ are $B-V\left(\tau^{-1}(0)\right.$.

Definition 3.1: Let $p: E \rightarrow B$ be a map of systems, where $\underline{E}=\left(E_{\lambda}, q_{\lambda \lambda_{i}}, A\right), \underline{B}=$ $\left(B_{\mu}, r_{\mu \mu_{1}}, M\right)$ and $\underline{p}=\left(p_{\mu}, \phi\right)$. We say $\underline{p}$ has the ASEP provided the following holds.

For each $(\lambda, \mu) \in A(\underline{p})$ and each $q_{\lambda} \in \operatorname{Cov} E_{\lambda}, \subset V_{\mu} \in \operatorname{Cov} B_{\mu}$, there exist $\left(\lambda_{1}, \mu_{1}\right) \geq(\lambda, \mu)$ in $A(\underline{p})$ and $\mathcal{V}_{\mu_{1}} \in \operatorname{Cov} B_{\mu_{1}}$ which satisfies the following:

(*) Suppose $\mu_{2} \geq \mu_{1}, V$ is a halo of a subset $A$ in $B_{\mu_{2}}$ and $s: V \rightarrow E_{\lambda_{1}}$ is a map with $\left(p_{\mu_{1} \lambda_{1}} s,\left.r_{\mu_{1} \mu_{2}}\right|_{V}\right) \leq \mathcal{V} \mu_{\mu_{1}}$. Then there exist $\mu_{3} \geq \mu_{2}$ and a map $S: B_{\mu_{3}} \rightarrow E_{\lambda}$ with $\left(p_{\mu \lambda} S, r_{\mu \mu_{3}}\right) \leq \mathcal{V}_{\mu}$ and $\left(\left.S\right|_{r_{\mu_{2} \mu_{3}}^{-1}(A)},\left.q_{\lambda \lambda_{1}} s r_{\mu_{2} \mu_{3}}\right|_{\mu_{2^{\mu_{3}}}} ^{-1}(A)\right) \leq \bigcup_{\lambda}$.

Proposition 3.2: Let $(\underline{q}, \underline{r}, \underline{p})$ and $\left(\underline{q}^{\prime}, \underline{r}^{\prime}, \underline{p}^{\prime}\right)$ be two AP-resolutions of a map $p: E \rightarrow B$. If $\underline{p}$ has the ASEP, then so does $\underline{p}^{\prime}$.

Note that under the notation of Proposition 1.1, all admissible pairs form an ANR (hence $A P$ )-resolution of the map $f$. Therefore by Proposition 1.1, the follhwing definition extends Definition 1.2.

Definition 3.3: Let $p: E \rightarrow B$ be a map. We say the map $p$ has the ASEP provided some (eq., any) AP-resolution of $p$ has the ASEP.

We can extend the concept of approximate invertibility ([A2]) by the same method.

Propositition And Definition 3.4: Suppose $p: E \rightarrow B$ be a map and $(\underline{q}, \underline{r}, \underline{p})$ is an AP-resolution of $p$, where $\underline{q}=\left(q_{\lambda}\right): E \rightarrow \underline{E}=\left(E_{\lambda}, q_{\lambda \lambda_{1}}, \Lambda\right), \underline{r}=\left(r_{\mu}\right): B \rightarrow \underline{B}=$ $\left(B_{\mu}, r_{\mu \mu_{1}}, M\right)$ and $\underline{p}=\left(p_{\mu}\right): \underline{E} \rightarrow \underline{B}$. Then the following conditions are equivaient and depend only on the map $p$.

(1) For each $(\lambda, \mu) \in A(\underline{p})$ and each $\mathcal{V}_{\mu} \in \operatorname{Cov} B_{\mu}$, there exist $\mu_{1} \geq \mu$ in $M$ and $a$ 
map $S: B_{\mu_{1}} \rightarrow E_{\lambda}$ with $\left(p_{\mu \lambda} S, r_{\mu \mu_{1}}\right) \leq \mathcal{V}_{\mu}$.

(2) For each $(\lambda, \mu) \in A(\underline{p})$ and each $\subset_{\nu_{\mu}} \in \operatorname{Cov} B_{\mu}$, there exists $S: B \rightarrow E_{\lambda}$ with $\left(p_{\mu \lambda} S, r_{\mu}\right) \leq \mathcal{V}_{\mu}$.

We say the map $p$ has approximate sections when the above conditions are satisfied.

REMARK 3.5: T. Watanabe also introduced ([W], Section 24) the concept of weak approximative dominations, which is essentially the same as ours.

RemmARK 3.6: 1) Every ANR-resolution of a space $X$ induces an HPolexpansion of $X$ in the category pro-HTop. Therefore by the definition, if a map $p: E \rightarrow B$ has approximate sections then $p$ induces a weak domination in Shape category.

2) Let $f: X \rightarrow Y$ be a map between metric spaces. If the map $f$ has approximate sections, then $f$ has a dense image.

The proof of 3.2 is similar to those of [Ma], Theorem 4, or [MR], Theorem 1 . For the sake of completeness, we give a full detail of the proof. As for notations, let $\underline{q}=\left(q_{\lambda}\right): E \rightarrow \underline{E}=\left(E_{\lambda}, q_{\lambda \lambda_{1}}, \Lambda\right), \underline{r}=\left(r_{\mu}\right): B \rightarrow \underline{B}=\left(B_{\mu}, r_{\mu \mu_{1}}, M\right), \underline{p}=\left(p_{\mu}\right): \underline{E} \rightarrow \underline{B}, \underline{q}^{\prime}=\left(q_{*}^{\prime}\right):$ $E \rightarrow \underline{F}=\left(F_{\kappa}, q_{\kappa k_{1}}^{\prime}, \bar{K}\right), \quad \underline{r}^{\prime}=\left(r_{\nu}^{\prime}\right): B \rightarrow \underline{C}=\left(C_{\nu}, r_{\nu \nu_{1}}^{\prime}, N\right), \underline{p^{\prime}}=\left(p_{\nu}^{\prime}\right): \underline{F} \rightarrow \underline{C}$. We need the following lemma. See [Ma], Definition 3 and [MR], Theorem 1 .

LEMMA 3.7: Under the above notation:

(1) For each $(\kappa, \nu) \in A\left(\underline{p}^{\prime}\right), \mathscr{W} \in \operatorname{Cov} F_{\kappa}$ and $\mathscr{Z} \in \operatorname{Cov} C_{\nu}$, there exist $(\lambda, \mu) \in A(\underline{p})$ and maps $f: E_{\lambda} \rightarrow F_{\kappa}, g: B_{\mu} \rightarrow C_{\nu}$ with $\left(f q_{\lambda}, q_{\kappa}^{\prime}\right) \leq \mathscr{W},\left(g r_{\mu}, r_{\nu}^{\prime}\right) \leq \mathscr{Z}$ and $\left(p_{\nu \kappa}^{\prime} f, g p_{\mu \lambda}\right) \leq \mathscr{Z}$.

(2) For each $(\kappa, \nu) \in A\left(\underline{p}^{\prime}\right), \mathscr{W} \in \operatorname{Cov} F_{\kappa}$ and $\mathscr{Z} \in \operatorname{Cov} C_{\nu}$, there exist $\mathscr{W} \mathcal{W}^{\prime} \in \operatorname{Cov} F_{\kappa}$ and $\mathscr{Z}^{\prime} \in \operatorname{Cov} C_{\nu}$ which refine $\mathscr{W}$ and $\mathscr{Z}$ resp. and satisfy the following:

For each $(\lambda, \mu) \in A(\underline{p})$ and maps $f: E_{\lambda} \rightarrow F_{k}, g: B_{\lambda} \rightarrow C_{\nu}$ with $\left(f q_{\lambda}, q_{k}^{\prime}\right) \leq \mathscr{W}{ }^{\prime},\left(g r_{\mu}, r_{\nu}^{\prime}\right)$ $\leq \mathscr{Z}^{\prime}$ and $\left(p_{\nu x}^{\prime} f, g p_{\mu \lambda}\right) \leq \mathscr{Z}^{\prime}$ and for each $\left(\lambda_{1}, \mu_{1}\right) \geq(\lambda, \mu)$ in $A(\underline{p})$ and $\mathcal{U}_{1} \in \operatorname{Cov} E_{\lambda_{1}}, \mathcal{V}_{1}$ $\in \operatorname{Cov} B_{\mu_{1}}$, there exist $\left(\kappa_{1}, \nu_{1}\right) \geq(\kappa, \nu)$ in $A\left(\underline{p}^{\prime}\right)$ and two maps $f_{1}: F_{\kappa_{1}} \rightarrow E_{\lambda_{1}}, g_{1}: C_{\nu_{1}} \rightarrow B_{\mu_{1}}$ with $\left(f_{1} q_{\kappa_{1}}^{\prime}, q_{\lambda_{1}}\right) \leq \mathcal{U}_{1}, \quad\left(g_{1} r_{\nu_{1}}^{\prime}, r_{\mu_{1}}\right) \leq \mathcal{V}_{1}, \quad\left(p_{\mu_{1} \lambda_{1}} f_{1}, g_{1} p_{\nu_{1} \kappa_{1}}^{\prime}\right) \leq \mathcal{V}_{1} \quad$ and $\quad\left(f q_{\lambda_{1}} f_{1}, q_{\kappa \kappa_{1}}^{\prime}\right) \leq \mathscr{W}$, $\left(g r_{u \mu_{1}} g_{1}, r_{\nu \nu_{1}}^{\prime}\right) \leq \mathscr{Z}$.

Proof of 3.2: The proof consists of the construction of the following diagram by Lemma 3.7 and the ASEP of $\underline{p}$. 


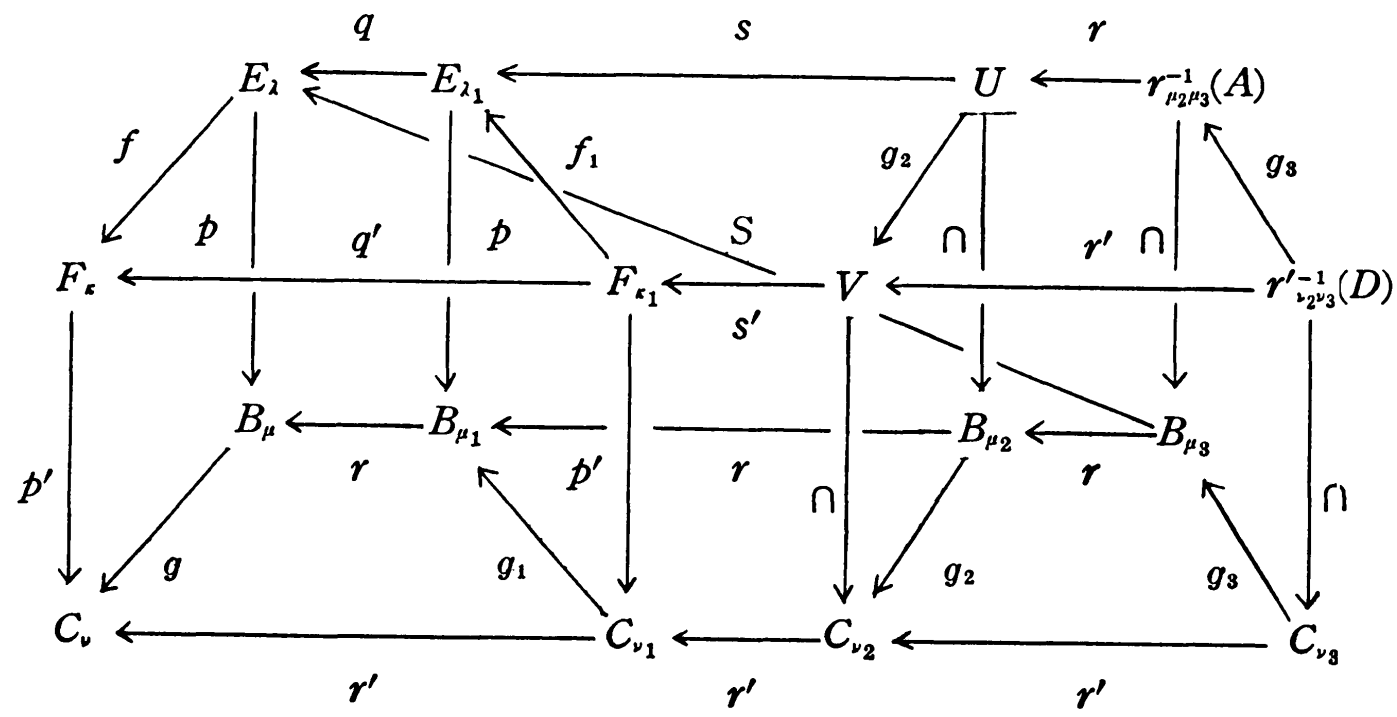

To show $\underline{p}^{\prime}$ has the ASEP, given $(\kappa, \nu) \in A\left(p^{\prime}\right), W_{\kappa} \in \operatorname{Cov} F_{\kappa}$ and $\mathscr{L}_{\nu} \in \operatorname{Cov} C_{\nu}$. Take $\mathscr{W}^{\prime} \in \operatorname{Cov} F_{x}$ and $\mathscr{L}^{\prime} \in \operatorname{Cov} C_{\nu}$ with st $\mathscr{W}^{\prime}<W_{k}$, st ${ }^{2} \mathscr{Z}^{\prime}<\mathscr{Z}_{\nu}$. Apply 3.7, (2) to $(\kappa, \nu), \mathcal{W}^{\prime}, \mathscr{Z}^{\prime}$ and we obtain

(a): $\mathcal{W}_{*}^{\prime} \in \operatorname{Cov} F_{x}$ and $\mathscr{L}_{\nu}^{\prime} \in \operatorname{Cov} C_{\nu}$ as in $3.7,(2)$.

By 3.7, (1), there exist $(\lambda, \mu) \in A(\underline{p})$ and maps $f: E_{\lambda} \rightarrow F_{\kappa}, g: B_{\mu} \rightarrow C_{\nu}$ with $\left(f q_{\lambda}, q_{\kappa}^{\prime}\right)$ $\leq W_{s}^{\prime},\left(g r_{\mu}, r_{\nu}^{\prime}\right) \leq \mathscr{Z}_{\nu}^{\prime},\left(p_{\nu \kappa}^{\prime} f, g p_{\mu \lambda}\right) \leq \mathscr{Z}_{\nu}^{\prime}$. Since $p$ has the ASEP, there exist

(b): $\left(\lambda_{1}, \mu_{1}\right) \geq(\lambda, \mu)$ and $\mathcal{V}_{\mu_{1}} \in \operatorname{Cov} B_{\mu_{1}}$ which satisfy 3.1 , (*) w. r.t. $(\lambda, \mu), f^{-1}\left(\mathscr{W}^{\prime}\right)$ $\epsilon \operatorname{Cov} E_{\lambda}$ and $g^{-1}\left(\mathcal{Z}^{\prime}\right) \in \operatorname{Cov} B_{\mu}$.

Take $C V_{\mu_{1}}^{\prime} \in \operatorname{Cov} B_{\mu_{1}}$ with st $C{\mathcal{\mu _ { 1 }}}^{\prime}<\mathcal{V}_{\mu_{1}} \wedge\left(g r_{\mu_{\mu_{1}}}\right)^{-1}\left(\mathscr{Z}^{\prime}\right)$. By 3.7, (2) (in this case, we apply the lemma only to $q$ and $q^{\prime}$ ), there exists

(c): $C V_{\mu_{1}}^{\prime \prime} \in \operatorname{Cov} B_{\mu_{1}}$ as in 3.7, (2) w. r.t. $\mu_{1}$ and $C V_{\mu_{1}}^{\prime}$.

We apply (a) to the data $(\lambda, \mu), f, g,\left(\lambda_{1}, \mu_{1}\right)$ and $\mathcal{V}_{\mu_{1}}^{\prime \prime} \in \operatorname{Cov} B_{\mu_{1}}$ to get $\left(\kappa_{1}, \nu_{1}\right) \geq$ $(\kappa, \nu)$ in $A(\underline{p})$ and maps $f_{1}: F_{\kappa_{1}} \rightarrow E_{\lambda_{1}}$ and $g_{1}: C_{\nu_{1}} \rightarrow B_{\mu_{1}}$ with $\left(g_{1} r_{\nu_{1}}^{\prime}, r_{\mu_{1}}\right) \leq C \mathcal{V}_{\mu_{1}}^{\prime \prime},\left(p_{\mu_{1} \lambda_{1}} f_{1}\right.$, $\left.g_{1} p_{\nu_{1} \kappa_{1}}^{\prime}\right) \leq C \mathcal{V}_{\mu_{1}}^{\prime \prime}$ and $\left(f q_{\lambda \lambda_{1}} f_{1}, q_{\kappa \kappa_{1}}^{\prime}\right) \leq \mathcal{W}^{\prime},\left(g r_{\mu \mu_{1}} g_{1}, r_{\nu \nu_{1}}^{\prime}\right) \leq \mathscr{Z}^{\prime}$.

Claim: $\left(\kappa_{1}, \nu_{1}\right)$ and $g_{1}^{-1}\left(C V_{\mu_{1}}^{\prime}\right) \in \operatorname{Cov} C_{\nu_{1}}$ satisfy $3.1,\left(^{*}\right)$ w.r.t. $(\kappa, \nu), W_{*}$ and $\mathscr{Z}_{\nu}$.

To see this, suppose $\nu_{2} \geq \nu_{1}$ in $N, V$ is an open halo of a subset $D$ in $C_{\nu_{2}}$ and $s^{\prime}: V \rightarrow F_{\kappa_{1}}$ is a map with $\left(p_{\nu_{1} \kappa_{1}}^{\prime} s^{\prime}, r_{\nu_{1} \nu_{2}}^{\prime} \mid V\right) \leq g_{1}^{-1}\left(C V_{\mu_{1}}^{\prime}\right)$. Take a haloing function $\tau: C_{\nu_{2}}$ $\rightarrow[0,1]$ with $D \subset \tau^{-1}(1)$ and $C_{\nu_{2}}-V \subset \tau^{-1}(0)$. Let $V_{1}=\tau^{-1}(1 / 2,1]$. Then $V$ is a halo of $V_{1}, \bar{D} \subset V_{1}$, and $r_{\nu_{2}}^{\prime-1}\left(\mathscr{Z}^{\prime}\right),\left\{C_{\nu_{2}}-\bar{D}, V_{1}\right\},\left(q_{\kappa_{1}}^{\prime} s^{\prime}\right)^{-1}\left(\mathcal{W}^{\prime}\right) \cup\left\{C_{\nu_{2}}-\bar{D}\right\} \in \operatorname{Cov} C_{\nu_{2}}$. Ta.ke $\mathscr{Z}_{\nu_{2}}$ $\in \operatorname{Cov} C_{\nu_{2}}$ which refines the above three coverings. Apply 3.7, (2) to $\nu_{2}$ and $\mathscr{Z}_{\nu_{2}}$, we obtain

(d): $\mathscr{L}_{\nu_{2}}^{\prime} \in \operatorname{Cov} C_{\nu_{2}}$ as in 3.7, (2).

We apply (c) to the data $\nu_{1}, g_{1}, \nu_{2}, \mathscr{Z}_{\nu_{2}}^{\prime}$ and obtain $\mu_{2} \geq \mu_{1}$ and a map $g_{2}: B_{\mu_{2}} \rightarrow$ $C_{\nu_{2}}$ with $\left(g_{2} r_{\mu_{2}}, r_{\nu_{2}}^{\prime}\right) \leq \mathscr{Z}_{\nu_{2}}^{\prime}$ and $\left(g_{1} r_{\nu_{1} \nu_{2}}^{\prime} g_{2}, r_{\mu_{1} \mu_{2}}\right) \leq C V_{\mu_{1}}^{\prime}$. Let $U=g_{2}^{-1}(V), A=g_{2}^{-1}\left(V_{1}^{\prime}\right)$ and $s=\left.f_{1} s^{\prime} g_{2}\right|_{U}: U \rightarrow E_{\lambda_{1}}$. Then $U$ is a halo of $A$ in $B_{\mu_{2}}$ and $\left(p_{\mu_{1} \lambda_{1}} s,\left.r_{\mu_{1} \mu_{2}}\right|_{U}\right) \leq C V_{\mu_{1}}$. 
Therefore (b) gives $\mu_{3} \geq \mu_{2}$ and a map $S: B_{\mu_{3}} \rightarrow E_{\lambda}$ with $\left(p_{\mu \lambda} S, r_{\mu \mu_{3}}\right) \leq g^{-1}\left(\mathscr{Z}^{\prime}\right)$ and $\left(\left.S\right|_{r_{\mu_{2} \mu_{3}}^{-1}(A)}, q_{\lambda_{1}} s r_{\mu_{2} \mu_{3}} \mid r_{\mu_{2} \mu_{3}}^{-1}(A)\right) \leq f^{-1}\left(\mathcal{W}^{\prime}\right)$. Apply (d) to $\mu_{2}, g_{2}, \mu_{3}$, and we get $\nu_{3} \geq \nu_{2}$ and a map $g_{3}: C_{\nu_{3}} \rightarrow B_{\mu_{3}}$ with $\left(g_{2} r_{\mu_{2} \mu_{3}} g_{3}, r_{\nu_{2} \nu_{3}}^{\prime}\right) \leq \mathscr{Z}_{\nu_{2}}$. Let $S^{\prime}=f S g_{3}: C_{\nu_{3}} \rightarrow F_{\kappa}$. Then adjacent maps of the followings are $\mathscr{Z}^{\prime}$-near:

$$
p_{\nu \kappa}^{\prime} S^{\prime}, g p_{\mu \lambda} S g_{3}, g r_{\mu \mu_{3}} g_{3}, g r_{\mu \mu_{1}} g_{1} r_{\nu_{1} \nu_{2}}^{\prime} g_{2} r_{\mu_{2} \mu_{3}} g_{3}, r_{\nu \nu_{2}}^{\prime} g_{2} r_{\mu_{2} \mu_{3}} g_{3}, r_{\nu \nu_{3}}^{\prime}
$$

This implies $\left(p_{\nu x}^{\prime} S^{\prime}, r_{\nu \nu_{3}}^{\prime}\right) \leq \mathscr{Z}_{\nu}$.

It remains only to show $\left(\left.q_{\kappa_{1}}^{\prime} s^{\prime} r_{\nu_{2} \nu_{3}}^{\prime}\right|_{\nu_{\nu_{2} \nu_{3}}^{\prime}(D)} ^{\prime-1},\left.S^{\prime}\right|_{r_{\nu_{2} \nu_{3}}^{\prime-1}(D)}\right) \leq \mathcal{W}_{\kappa}$. First note that $g_{3}\left(r_{\nu_{2} \nu_{3}}^{\prime-1}(D)\right) \subset r_{\mu_{2} \mu_{3}}^{-1}(A)$ and that, since $\left(g_{2} r_{\mu_{2} \mu_{3}} g_{3}, r_{\nu_{2} \nu_{3}}^{\prime}\right) \leq\left(q_{\kappa_{1}}^{\prime} s^{\prime}\right)^{-1}\left(\mathcal{W}^{\prime}\right) \cup\left\{C_{\nu_{2}}-\bar{D}\right\}$, maps $g_{2} r_{\mu_{2} \mu_{3}} g_{3}, r_{\nu_{2} \nu_{3}}^{\prime}: r_{\nu_{2} \nu_{3}}^{\prime-1}(D) \rightarrow V$ are $\left(q_{\kappa \kappa_{1}}^{\prime} s^{\prime}\right)^{-1}\left(\mathcal{W}^{\prime}\right)$-near. Thus adjacent maps of the followings are $\mathscr{W}^{\prime}$-near on $r_{2^{2} 3}^{\prime-1}(D)$ :

$$
q_{\kappa \kappa_{1}}^{\prime} s^{\prime} r_{\nu_{2} \nu_{3}}^{\prime}, q_{\kappa_{1}}^{\prime} s^{\prime} g_{2} r_{\mu_{2} \mu_{3}} g_{3}, f q_{\lambda_{1} \lambda_{1}} s r_{\mu_{2} \mu_{3}} g_{3}, S^{\prime}
$$

Since st $\mathscr{W}^{\prime}<\mathscr{W}_{\kappa}$, we have the conclusion.

We conclude the section, inspecting Taylor's map $F: X \rightarrow \boldsymbol{Q}$ ([DW], [T]).

EXAMPLE 3.8 (Taylor's map): The map $F$ is obtained as the inverse limit of the following level map $\underline{f}=\left\{f_{n}\right\}$ :

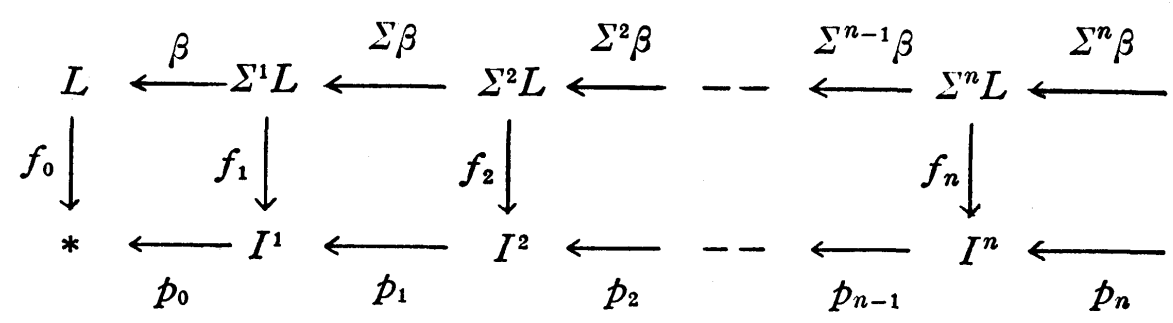

where $I=[-1,1]^{r}$ ( $r$ is a fixed positive integer), $\Sigma^{n} L$ is the $n r$-th suspension of a compact polyhedron $L$ and $\beta$ is a map for which the composition $\beta \circ \Sigma \beta \circ \cdots \circ \Sigma^{n} \beta \neq 0$ for each $n \geq 0, f_{n}$ is induced from the projection $L \times I^{n} \rightarrow I^{n}$ and $p_{n}$ is the projection. Note that, by [Ma], Theorem $8, \underline{f}$ is an ANR-resolution of $F$. The map $F$ is an example of CE-maps which are not shape equivalences $[\mathrm{TT}]$, and moreover, $F$ is approximately invertible ([A2]). Note that each $f_{n}$ has a section.

We now show that $F$ does not have the ASEP. To see this, on the contrary, suppose $F$, hence $\underline{f}$ has the ASEP. Then there exist $n \geq 0$ and $\varepsilon>0$ which satisfy 3.1, (*) w.r. t. the index 0 in $\underline{f}$. Since $\Sigma^{n} L$ is a finite dimensional compact metric space, we can find an embedding $i: \Sigma^{n} L \rightarrow I^{m}$ for some $m \geq 0$. Define an embedding $j: \Sigma^{n} L \rightarrow I^{n_{+} m}=I^{n} \times I^{m}$ by $j(x)=\left(f_{n}(x), i(x)\right)\left(x \in \Sigma^{n} L\right)$ and let $A=j\left(\Sigma^{n} L\right), s=j^{-1}: A \rightarrow$ $\Sigma^{n} L$. Since $f_{n} s=\left.p_{n n+m}\right|_{A}$, by the choice of $n$, there exist $k \geq n+m$ and a map $S$ : $I^{k} \rightarrow L$ such that $S$ coincides with the composition $\beta \circ \cdots \circ \sum^{n-1} \beta \circ S \circ p_{n+m, k}$ on $p_{n+m, k}^{-1}(A)$ $=A \times I^{k-n-m}$. Since $S \simeq 0$ and $s p_{n+m, k}: A \times I^{k-n-m} \rightarrow \Sigma^{n} L$ is a homotopy equivalence, 
$\beta \circ \cdots \circ \Sigma^{n-1} \beta \simeq 0$. This contradicts the choice of $\beta$.

One can embeds $X$ into $\boldsymbol{Q}$ and extend $F$ to the CE-map $G: \boldsymbol{Q} \rightarrow \boldsymbol{Q} \cup_{F} \boldsymbol{Q}$. It is known that $\boldsymbol{Q} \cup{ }_{F} \boldsymbol{Q}$ does not have the trivial shape ([DS]), hence $G$ is not a weak domination. This implies that $G$ is not approximately invertible.

\section{References}

[A1] Ancel, F.D., The role of countable dimensionality in the theory of cell-like relations, Trans. Amer. Math. Soc. (to appear).

[A2] - Approximate countable dimensionality and cell-like maps, in preparation.

[DW] Daverman, R. J. and Walsh, J. J., Examples of cell-like maps that are not shape equivalences, Michigan Math. J. 30 (1983) 17-30.

[Do] Dold, A., Partitions of unity in the theory of fibrations, Annals of Math. "78 (1963) 223-255.

[Dy] Dydak, J., The Whitehead and the Smale theorems in shape theory, Dissertationes Math. 156.

[DS] and Segal, J., Shape theory, Lecture Note in Math. Vol. 688, SpringerVerlag, New York, 1978.

[H] Hu, S.T., Theory of Retracts, Wayne State Univ. Press, 1965.

[K] Kozlowski, G., Images of ANR's, Trans. Amer. Math. Soc. (to appear).

[L] Lacher, R. C., Cellularity criteria for maps, Michigan Math. J. 17 (1970) 385-396.

[Ma] Mardešis, S., Approximate polyhedra, resolution of maps and shape fibrations, Fund. Math. 114 (1981) 53-78.

[MR] - and Rushing, T. B., Shape fibrations I, Gen. Top. and its Appli. 9 (1978) 193-215.

[Mi] Michael, E., Local properties of topological spaces, Duke Math. J. 21 (1954) 163-172.

[T] Taylor, J. L., A counterexample in shape theory, Bull. Amre. Math. Soc. \&1 (1975) 629-632.

[W] Watanabe, T., Approximative shape theory, in preparation.

Institute of Mathematics

University of Tsukuba

Sakuramura, Ibaraki, 305 Japan. 